\title{
Fabrication, electrochemical and electrocatalytic properties of carbon nanotube@nano-SiO 2 BenV/phosphomolybdic acid polynary nanocomposite materials
}

\author{
Jiang Liu, ${ }^{a}$ Jing Wang, ${ }^{a}$ Wen-Bo Wang, ${ }^{a}$ Meng Chen, ${ }^{b}$ Dong-Jin Qian ${ }^{a^{*}}$ \\ ${ }^{a}$ Department of Chemistry, Fudan University, 220 Handan Road, Shanghai 200433, China \\ ${ }^{b}$ Department of Material Science, Fudan University, 220 Handan Road, Shanghai 200433,
}

China

\section{Abstract}

Organic-inorganic nano-materials have attracted growing attention due to their potential applications for optoelectronic devices, sensors, and heterogeneous catalysts. We reported here on the preparation of polynary nanocomposites composed of poly(4-vinylpyridine) (P4VP) functionalized multi-walled carbon nanotubes (MWNTP4VP), silica nanoparticles (nano-SiO 2 ), viologens, and/or phosphomolybdic acid (PMA), in which the MWNTP4VP, nano-SiO 2 , and viologens were covalently connected while PMA was electrostatically adsorbed. Thermogravimetric analysis revealed that the nanocomposites were composed of about 40-45\% MWNTs, 40-45\% nanoSiO 2 , as well as 10-15\% organic species and others. The preparation processes and compositions of the nanocomposites were characterized using Fourier transform infrared spectroscopy and X-ray photoelectron spectroscopy. Field emission transmission electron microscopic images revealed that the nano- $\mathrm{SiO}_{2} \mathrm{BenV}$ particles were strongly attached 
to the MWNTP4VP surfaces to form MWNTP4VP@nano-SiO ${ }_{2} \mathrm{BenV}$ triad nano-cores. Cyclic voltammograms of the MWNTP4VP@nano-SiO ${ }_{2} \mathrm{BenV}$ casting films showed three couples of redox waves in the potential range between -0.8 and $0 \mathrm{~V}$ ( $\mathrm{vs} \mathrm{Ag} / \mathrm{AgCl}$ ), designated to the electron transfer process of viologen substituents of MWNTP4VP@nano-SiO $\mathrm{BenV}^{2+} \leftrightarrow$ MWNTP4VP@nano-SiO ${ }_{2} \mathrm{BenV}^{+\bullet}$ and their dimers. Further, three couples of redox waves were recorded for the casting films of MWNTP4VP@ nano-SiO 2 BenV/PMA polynary nanocomposites in the potential range between -0.2 and $0.8 \mathrm{~V}$, designated to three successive electron transfer processes of PMA. Finally, it was revealed that the present MWNTPVP@nano-SiO 2 BenV/PMA polynary composites could act as efficient heterogeneous catalysts for the electrocatalytic reduction of bromate.

Keywords: Nano-composites; Carbon nanotube; Viologen; Phosphomolybic acid;

Electrochemistry; Electrocatalysis

* Corresponding author

E-mail: djqian@fudan.edu.cn

Tel: +86-21-65643666

\section{Introduction}

Carbon nanotubes (CNTs) have one-dimensional (1D) architecture with unique electronic, thermal, and mechanical properties. Since their discovery, they have shown many potential applications for the development of novel composite/hybrid materials [1], sensors [2], sensitized 
solar cells [3], and nanoscale electronic components [4,5]. To realize these potential applications, it is necessary to modify CNTs to produce their functional nanohybrids and nanocomposites, which can improve their dispersion in the solutions, introduce organic substituents on their surfaces, and enhance their optical, electrical, and magnetic properties [6-8]. In the past decades, many organic, inorganic, and polymeric substituents have been anchored on CNT surfaces by covalent or noncovalent molecular interactions $[9,10]$. Here, we reported a strategy to covalently anchor nanoscale $\mathrm{SiO}_{2}$ particles on the surfaces of CNTs to form 1D CNT@ nano-SiO $\mathrm{S}_{2}$ inorganic nano-cores that further acted as a support to construct 1D organic-inorganic polynary composite materials with viologens and/or polyoxomelates (as a "shell") based on covalent binding and electrostatic interactions.

It is well known that silica has the advantages of a low production cost, mechanical and chemical stability, and optical transparency. The surface of silica is rich in hydroxyl groups, which renders it intrinsically hydrophilic, favorable for colloidal stability, and easily modifiable by various functional moieties via well-established siloxane chemistry to gain multi-functional materials [11]. The combination of CNTs with silica is expected to further increase the possibility of modifying $\mathrm{CNT}-\mathrm{SiO}_{2}$ composites with a large variety of functional groups, of thermal and chemical stability, of a larger surface active area, and particularly of electrical conductivity [12-15].

An earlier study by Liu and coworkers reported growth of a thin layer of $\mathrm{SiO}_{2}(\sim 1 \mathrm{~nm})$ on the sidewalls of single-walled CNTs (SWNTs) using 3-aminopropyltriethoxysilane as coupling layers [16]. Wojdel and Bromley predicted that silica can provide a protective bound coating for SWNTs and only weakly perturb the underlying properties of both components based on a periodic density functional calculation [17]. A method was then developed by Wang and 
coworkers, wherein a well-defined silica-coated CNT coaxial nano-composite was prepared by a combination of sonication with a sol-gel technique without prior CNT functionalization [18]. Further, the silica/CNT nano-composite was used as a 3D support for loading ultra-high-density metal or hybrid nanoparticles (NPs) including gold NPs, Au/Pt NPs to produce enhanced materials for the construction of high-performance electrochemical devices. For instance, the detection limit of the NP-modified nano-composites for $\mathrm{H}_{2} \mathrm{O}_{2}$ was found to be $0.3 \mu \mathrm{M}$; lower than certain enzyme-based biosensors. The $\mathrm{CNT} / \mathrm{SiO}_{2} /(\mathrm{Au} / \mathrm{Ag})$ nano-composites showed great potential as surface-enhanced Raman scattering substrate for biological tags and biomolecular molecular detection. More recently, the $\mathrm{CNT} / \mathrm{SiO}_{2} / \mathrm{Au} \mathrm{NP}$ nano-composites were developed as a label for the electrochemical immunoassay of carcinoembryonic antigen in biological fluids [19]. Other reports on the preparation and applications of the $\mathrm{CNT} / \mathrm{SiO}_{2}$ nano-composites included a covalent interaction between chlorosilane and acid-treated CNTs as well as non-covalent molecular interactions between functionalized CNTs and organic-inorganic hybrids for the construction of superhydrophobic conductive coatings and the development of core-shell catalysts, electrochemiluminescence sensors, ultrahigh lithium storage, and others [20-24].

Herein, our strategy contained a three-step process to prepare MWNTP4VP@nano-SiO $\mathrm{O}_{2} \mathrm{BenV}$ and MWNTP4VP@nano-SiO 2 BenV/polyoxomelate polynary composite materials. The first step was the covalent binding of multi-walled carbon nanotubes (MWNTs) with poly(4-vinylpyridine) (P4VP) to form the P4VP-functionalized MWNTP4VP hybrids and the covalent binding of nano- $\mathrm{SiO}_{2}$ particles with (4-chloromethylphenyl)trimethoxylsilane to form benzyl chloride functionalized nano- $\mathrm{SiO}_{2} \mathrm{BenCl}$ hybrids (Fig. 1). The second step was the other covalent combination of the MWNTP4VP hybrids with the nano- $\mathrm{SiO}_{2} \mathrm{BenCl}$ ones to produce the MWNTP4VP@nano-SiO 2 BenCl nano-cores. Finally, electroactive viologens (Vs) and 
phosphomolybdic acid (PMA) were covalently and electrostatically bounded on the surfaces of the MWNTP4VP@nano- $\mathrm{SiO}_{2} \mathrm{BenCl}$ nano-cores to produce the polynary composites. Electrochemical investigations revealed that the present MWNTP4VP@nano-SiO ${ }_{2} \mathrm{BenCl} / \mathrm{PMA}$ composite materials are well-stable electroactive materials and efficient heterogeneous catalysts for the electrocatalytic reduction of bromate.

\section{Experimental section}

\subsection{Materials}

MWNTs (diameter 10-20 nm, purity >95\%) were purchased from Chengdu Organic Chemicals Co. Ltd., Chinese Academy of Sciences. P4VP, 4-(hydroxymethyl) aniline, 4,4'bipyridyl, and (4-chloromethylphenyl)trimethoxylsilane were from Sigma-Aldrich Co. Ludox AS40, obtained from Aldrich, was used as the starting silica material. It contains $40 \mathrm{wt} \% \mathrm{SiO}_{2}$, with an average particle size of $12 \mathrm{~nm}$. All chemicals were used as received without further purification.

\subsection{Preparation of $1 D$ MWNTP4VP@nanoSiO ${ }_{2}$ BenV/PMA nano-composites}

Fig. 1 shows a schematic representation of the preparation of the present 1D nano-composites, which can be divided into the following steps: (i) the functionalization of MWNTs as-received with P4VP to produce MWNTP4VP hybrids [25], (ii) the preparation of benzyl chloride functionalized silica nanoparticles of nano- $\mathrm{SiO}_{2} \mathrm{BenCl}$ hybrids [26], and (iii) the construction of 
MWNTP4VP@nano-SiO ${ }_{2} \mathrm{BenCl}$ nano-cores, as well as MWNTP4VP@nano-SiO ${ }_{2} \mathrm{BenV}$ and MWNTP4VP@nano-SiO2BenV/PMA nano-composites.

More specifically, the MWNTP4VP hybrids were first prepared via the diazonium process followed by refluxing with P4VP in methanol solution (Fig. 1, A), as described in our previous work [25].

Figure 1

Second, the benzyl chloride functionalized silica nanoparticles of nano- $\mathrm{SiO}_{2} \mathrm{BenCl}$ were prepared by a sol-gel process according to Ribeiro et al.'s method (Fig. 1, B) [26]. Briefly, Ludox silica sol (10 g) was first suspended in the mixed solvents of methanol and DMF (100 mL) and stirred with $0.4 \mathrm{~g}$ of (4-chloromethylphenyl)trimethoxylsilane at $60{ }^{\circ} \mathrm{C}$ for $48 \mathrm{hrs}$. Then, the suspension was isolated by centrifugation at 4,000 rpm. The white powders of nano- $\mathrm{SiO}_{2} \mathrm{BenCl}$ nanohybrids were washed thoroughly with plenty of DMF and methanol and then dried in a vacuum overnight.

Third, the MWNTP4VP@nano-SiO ${ }_{2} \mathrm{BenCl}$ nanocores were prepared by refluxing $20 \mathrm{mg}$ of MWNTP4VP and $20 \mathrm{mg}$ of nano-SiO${ }_{2} \mathrm{BenCl}$ hybrids in $30 \mathrm{~mL}$ of methanol solution for $48 \mathrm{hrs}$ (Fig. 1, C), the suspension of which was centrifuged at 4,000 rpm and washed thoroughly using methanol and dried in a vacuum.

Fourth, the MWNTP4VP@ nano-SiO 2 BenV nano-hybrids were prepared by stirring a mixture of $10 \mathrm{mg}$ of MWNTP4VP@nano-SiO ${ }_{2} \mathrm{BenCl}$ nano-cores and $100 \mathrm{mg}$ of N-methyl-N'bipyridinium iodide salt in methanol at $60{ }^{\circ} \mathrm{C}$ for $24 \mathrm{hrs}$ (Fig. 1, D). The product was isolated by centrifugation at 4,000 rpm, washed thoroughly with methanol and water and dried in a vacuum. 
Finally, the MWNTP4VP@nano-SiO 2 BenV/PMA nano-composites were prepared by stirring a mixture of $100 \mathrm{mg}$ of PMA and $10 \mathrm{mg}$ of MWNTP4VP@nano-SiO${ }_{2} \mathrm{BenV}$ in the methanol solution at room temperature overnight (Fig. 1, E). The solid product was collected by centrifugation at 4,000 rpm, washed thoroughly with plenty of water to remove unreacted PMA and finally dried in a vacuum.

As a control experiment, electroactive nano- $\mathrm{SiO}_{2} \mathrm{BenV}$ hybrids were prepared by stirring a mixture of $20 \mathrm{mg}$ of nano- $\mathrm{SiO}_{2} \mathrm{BenCl}$ and $100 \mathrm{mg}$ of $\mathrm{N}$-methyl-N'-bipyridinium iodide salt in methanol at $60{ }^{\circ} \mathrm{C}$ for $24 \mathrm{hrs}$ (Fig. 1, C-1). The product was isolated by centrifugation at 4,000 rpm, washed thoroughly with methanol and water, and dried in a vacuum.

\subsection{Characterization}

Thermogravimetric (TG) analysis was performed on an STDQ600 instrument (TA Ltd. USA) under a constant air flow atmosphere from room temperature to $800^{\circ} \mathrm{C}$ at a rate of $10^{\circ} \mathrm{C} / \mathrm{min}$. Fourier transform infrared spectra (FITR) were measured by using a Nicolet NEXUS 470 spectrometer, operating at a resolution of $0.5 \mathrm{~cm}^{-1}$ at $25^{\circ} \mathrm{C}$.

X-ray photoelectron spectra (XPS) were recorded using a VGESCALAB MKII multifunction spectrometer with nonmonochromatized $\mathrm{Mg}-\mathrm{K} \alpha \mathrm{X}$-rays as the excitation source. The system was carefully calibrated by the Fermi edge of nickel, $\mathrm{Au} 4 \mathrm{f}_{2 / 7}$, and $\mathrm{Cu} 2 \mathrm{p}_{2 / 3}$ binding energy. A pass energy of $70 \mathrm{eV}$ and a step size of $1 \mathrm{eV}$ were chosen when recording the spectra. In the analysis chamber, pressures of $1 \sim 2 \times 10^{-7} \mathrm{~Pa}$ were routinely maintained. The binding energies obtained in the XPS analysis were corrected by referencing the $\mathrm{C} 1 \mathrm{~s}$ peak to $284.60 \mathrm{eV}$. 
Field emission transmission electron microscopy (FETEM) images were recorded using a JEM2100F field emission electron microscope.

\subsection{Electrochemical and electrocatalytic measurements}

The cyclic voltammogram (CV) was measured using an electrochemical analyzer (CHI 601b, China). A Pt wire and an $\mathrm{Ag} / \mathrm{AgCl}$ electrode were used as the auxiliary and reference electrodes, respectively. The glass carbon (GC) substrate covered with the nanoSiO${ }_{2} \mathrm{BenV}$ hybrids and MWNTP4VP@nano-SiO ${ }_{2} \mathrm{BenV}$ nano-composites, was used as the working electrode with 0.05 $\mathrm{mol} / 1 \mathrm{KCl}$ as the electrolyte. An initial potential of -1.0 or $-0.8 \mathrm{~V}$ was applied for $2 \mathrm{~s}$, and subsequently cyclic scans to a final potential of -0.4 or $0 \mathrm{~V}$ were done for 10 cycles.

The nano- $\mathrm{SiO}_{2} \mathrm{BenV}$ and $\mathrm{MWNTP} 4 \mathrm{VP} @$ nano- $\mathrm{SiO}_{2} \mathrm{BenV}$ casting film was prepared by dispersing the nanomaterials in $0.2 \%$ Nafion solution. The GC working electrode was polished with 0.3 and $0.05 \mu \mathrm{m} \mathrm{Al}_{2} \mathrm{O}_{3}$ paste before coating. Then, $3 \mu \mathrm{l}$ of well-dispersed nanomaterials in the Nafion solution was dropped onto the GC surface and dried at room temperature.

Electrocatalytic experiments were performed by recording the CV curves with the casting films of MWNTP4VP@nano-SiO ${ }_{2} \mathrm{BenV} / \mathrm{PMA}$ nano-composites as the working electrode in the 1 $\mathrm{mol} / \mathrm{l} \mathrm{LiCl}$ electrolyte solution $(\mathrm{pH}=2)$. The bromate reduction reaction was carried out using different concentrations of $\mathrm{KBrO}_{3}$ from 1 to $5 \mathrm{mmol} / \mathrm{l}$ at a scan rate of $50 \mathrm{mV} / \mathrm{s}$. All electrochemical measurements were done under Ar atmosphere at room temperature. 


\section{Results and discussion}

\subsection{Preparation of ID MWNTP4VP@nano-SiO ${ }_{2}$ BenV/PMA nano-composites}

To obtain the present nanocomposites, the MWNTs were first functionalized with P4VP to produce the MWNTP4VP hybrids, and the nano- $\mathrm{SiO}_{2}$ particles were reacted with (4chloromethylphenyl)-trimethoxylsilane to produce the nano- $\mathrm{SiO}_{2} \mathrm{BenCl}$ hybrids. As shown in Fig. 1, the MWNTs as-received were modified with a highly active benzyl bromide intermediate (MWNTBenBr) via the diazonium coupling process [25,27], followed by an addition reaction with $\mathrm{P} 4 \mathrm{VP}$ to produce the MWNTP4VP hybrids. The nano- $\mathrm{SiO}_{2} \mathrm{BenCl}$ hybrids were obtained by a sol-gel process [26]. Then, two kinds of nano-hybrids were refluxed in methanol for 2 days to form 1D MWNTP4VP@nano-SiO ${ }_{2} \mathrm{BenCl}$ nano-cores.

The surface of the MWNTP4VP@nano- $\mathrm{SiO}_{2} \mathrm{BenCl}$ nano-cores was covered by highly active benzyl chloride substituents that can react with a variety of organic compounds, particularly those with pyridine substituents. Here, N-methyl-N'-bipyridinium salt was reacted with the MWNTP4VP@nano-SiO ${ }_{2} \mathrm{BenCl}$ nano-cores to produce electroactive viologen functionalized nano-composites of MWNTP4VP@nano- $\mathrm{SiO}_{2} \mathrm{BenV}$. Viologens are a kind of positively charged organic electrolyte and have been widely used as electron mediators and electrochromism in various systems for the development of (bio)sensors and molecular devices [28-30].

Finally, because the MWNTP4VP@nano- $\mathrm{SiO}_{2} \mathrm{BenV}$ nan-composites were of positively charged nanomaterials that can act as supports to adsorb negatively charged polyelectrolyte or clusters based on the intermolecular electrostatic interaction and van der Waals force, we further prepared the MWNTP4VP@nano-SiO 2 BenV/PMA multifunctional nanocomposites with PMA, a kind of well-known polyoxomelate that has both interesting electrochemical and 
electrocatalytic properties [31,32]. The nanomaterials as-prepared were characterized by using TG, FTIR, XPS, and FETEM techniques as described below.

\subsection{Thermogravimetric analysis}

Fig. 2 shows $\mathrm{TG}$ curves for the nanomaterials of nano- $\mathrm{SiO}_{2} \mathrm{BenCl}$, nano- $\mathrm{SiO}_{2} \mathrm{BenV}$, MWNTP4VP@nano-SiO ${ }_{2} \mathrm{BenCl}$, and MWNTP4VP@ nano-SiO ${ }_{2} \mathrm{BenV}$ from room temperature to $800{ }^{\circ} \mathrm{C}$ in air. A gradual reduction in the mass was recorded for the nano- $\mathrm{SiO}_{2} \mathrm{BenCl}$ and nano$\mathrm{SiO}_{2} \mathrm{BenV}$ hybrids until $660{ }^{\circ} \mathrm{C}$, with a total mass reduction of about $10 \%$ and $14 \%$, respectively. This could be attributed to the decomposition of the organic species attached on the nanoSiO surfaces. It could also be due to the condensation reaction of $2 \mathrm{Si}-\mathrm{OH} \rightarrow \mathrm{Si}-\mathrm{O}-\mathrm{Si}+\mathrm{H}_{2} \mathrm{O}$ on the surface of the nanoparticles resulting in the evaporation of the water molecules produced, though the relative amount of the water produced was quite lower than that of the organic species [33].

Figure 2

When the nano-SiO $2 \mathrm{BenCl}$ and nano- $\mathrm{SiO}_{2} \mathrm{BenV}$ hybrids were anchored on the MWNTP4VP surfaces to form the MWNTP4VP@nano-SiO ${ }_{2} \mathrm{BenCl}$ and MWNTP4VP@nano-SiO $\mathrm{BenV}_{2}$ composites, the TG curves revealed a gradual reduction from 200 to about $450{ }^{\circ} \mathrm{C}$ and then a quick one from 500 to $660{ }^{\circ} \mathrm{C}$. The former gradual reduction process could be attributed to the decomposition of the organic species and the formation of a Si-O-Si bond (evaporation of water produced); the latter one was attributed to the decomposition of carbon nanotubes, as frequently reported in the literature [34]. The total mass reduction was about 55 and $59 \%$ for the MWNTP4VP@nano-SiO ${ }_{2} \mathrm{BenCl}$ and MWNTP4VP@nano-SiO ${ }_{2} \mathrm{BenV}$ composites, respectively. 
The residuals were $\mathrm{SiO}_{2}$ solid powders produced at a high temperature. These data indicated that the nano-composites were composed of about 40-45\% MWNTs, 40-45\% nanoSiO 2 , as well as $10-15 \%$ organic species and others.

\subsection{Fourier transform infrared spectra}

Fig. 3 shows the FTIR spectra for the present nanomaterials of nano- $\mathrm{SiO}_{2} \mathrm{BenCl}$, nano$\mathrm{SiO}_{2} \mathrm{BenV}, \quad \mathrm{MWNTP} 4 \mathrm{VP} @$ nano-SiO${ }_{2} \mathrm{BenCl}$, and MWNTP4VP@nano-SiO ${ }_{2} \mathrm{BenV}$, which revealed the following features. First, a broad band was recorded at about $3450 \mathrm{~cm}^{-1}$ for both nanohybrids and nano-composites, which could be attributed to the $\mathrm{O}-\mathrm{H}$ groups on the surface of the nano- $\mathrm{SiO}_{2}$ particles and MWNTs $[35,36]$. Second, two sharp peaks were recorded at about 2924 and $2848 \mathrm{~cm}^{-1}$, which were assigned to the $\mathrm{C}-\mathrm{H}$ stretch vibrations of the methyl groups from benzyl chloride attached on the nanohybrids and/or from the P4VP substituents on the MWNTP4VP hybrids. The relative intensity of these peaks in the spectra of the nano-composites was stronger than that in the spectra of the nanohybrids because of the attachment of P4VP on the surfaces of the nano-composites. Third, there were several peaks at the wavelength numbers

from 1350 to $1650 \mathrm{~cm}^{-1}$, which was designated to the $\mathrm{C}-\mathrm{H}, \mathrm{C}=\mathrm{N}$, and $\mathrm{C}=\mathrm{C}$ vibrations of the pyridyl groups from benzyl chloride and P4VP. Finally, the vibration bands recorded between 1095 and $805 \mathrm{~cm}^{-1}$ could be ascribed to the $\mathrm{Si}-\mathrm{O}-\mathrm{Si}$ asymmetric and $\mathrm{Si}-\mathrm{O}$ symmetric stretching bands of the nanomaterials [36]. The relative intensity of these bands was much stronger than that of the others due to the higher contaminant of $\mathrm{SiO}_{2}$ in the products. Thus, these FTIR spectra confirmed the formation of the nano- $\mathrm{SiO}_{2} \mathrm{BenCl}$ and nano- $\mathrm{SiO}_{2} \mathrm{BenV}$ hybrids as well as the 
MWNTP4VP@nano-SiO ${ }_{2} \mathrm{BenCl}$ and MWNTP4VP@nano-SiO ${ }_{2} \mathrm{BenV}$ ones, as we designed in Fig. 1.

Figure 3

\subsection{X-ray photoelectron spectra}

The compositions of the present nano-materials were investigated with the use of the XPS technique. Table 1 summarizes the peak positions for the detected elements of the nano$\mathrm{SiO}_{2} \mathrm{BenCl}$ and nano-SiO${ }_{2} \mathrm{BenV}$ hybrids as well as the MWNTP4VP@nano-SiO ${ }_{2} \mathrm{BenCl}$, MWNTP4VP@nano-SiO ${ }_{2} \mathrm{BenV}$ and MWNTP4VP@nano-SiO ${ }_{2}$ BenV/PMA composites. As an example, the high-resolution XPS peaks of MWNTP4VP@nano-SiO ${ }_{2} \mathrm{BenV}$ composites are shown in Fig. 4. Based on the data in Table 1, peaks in Fig. 4, and the structure of the MWNTs and nano- $\mathrm{SiO}_{2}$ particles before and after functionalization, the following features can be deduced.

\section{Table 1}

Figure 4

First, $\mathrm{Si}, \mathrm{Cl}, \mathrm{C}$, and $\mathrm{O}$ were detected in the nano- $\mathrm{SiO}_{2} \mathrm{BenCl}$ hybrids, among which $\mathrm{Si}$ and $\mathrm{O}$ originated from the nano- $\mathrm{SiO}_{2}$ core while $\mathrm{C}$ and $\mathrm{Cl}$ were from the benzyl chloride substituent. When viologen was formed on the surface of the nano- $\mathrm{SiO}_{2} \mathrm{BenCl}$ hybrids to produce the nano$\mathrm{SiO}_{2} \mathrm{BenV}$ ones, $\mathrm{N}$ and I were detected; $\mathrm{N}$ was from the bipyridinium substituent and $\mathrm{I}$ was the counter ion. Secondly, Br, Si, Cl, C, N, and O were detected from the MWNTP4VP@nano$\mathrm{SiO}_{2} \mathrm{BenCl}$ nanocores, among which $\mathrm{C}$ (partly) and $\mathrm{O}$ were from the MWNTs, $\mathrm{Br}$ originated from the highly active benzyl bromide of MWNTBenBr, and N from P4VP; C also originated from the benzyl bromide and P4VP substituents. Thirdly, for the MWNTP4VP@nano-SiO ${ }_{2} \mathrm{BenV}$ 
nano-composites, another element of I was detected, which was the counter ion of the viologen substituent. Finally, seven elements of $\mathrm{Si}, \mathrm{P}, \mathrm{Cl}, \mathrm{Mo}, \mathrm{C}, \mathrm{N}$ and $\mathrm{O}$ were detected from the polynary MWNTP4VP@nano-SiO ${ }_{2}$ BenV/PMA composites. It can be found that, instead of $\mathrm{Br}$ and I, the elements of $\mathrm{P}$ and Mo were detected, which indicated that the smaller anionic ions of $\mathrm{Br}^{-}$and $\mathrm{I}^{-}$were replaced by the larger PMA clusters in the polynary composites just as we designed in Fig. 1. Thus, the XPS data confirmed further formation of the present nano-hybrids and nanocomposites, as designed in Fig. 1.

\subsection{Morphological characterization}

Fig. 5 shows the FETEM images for the nano- $\mathrm{SiO}_{2}$ particles; the nano- $\mathrm{SiO}_{2} \mathrm{BenCl}$, nano$\mathrm{SiO}_{2} \mathrm{BenV}$, and MWNTP4VP hybrids; the MWNWP4VP@nano-SiO ${ }_{2} \mathrm{BenCl}$ nano-cores; and the MWNTP4VP@nano-SiO ${ }_{2} \mathrm{BenV}$ nanocomposites. First, the image of nano-SiO $\mathrm{Sarticles}_{2}$ suggested that these nanoparticles easily form aggregates before being functionalized with the organic species (Fig. 5A). One possible reason may be attributed to the fact that the commercial nano- $\mathrm{SiO}_{2}$ particles are stabilized in an aqueous solution with a $\mathrm{pH}$ of 9.8 , so their surfaces are covered with plenty of hydroxyl groups that result in a strong hydrogen bonding and hydrophilic interactions. When the organic $-\mathrm{BenCl}$ or $-\mathrm{BenV}$ substituents are modified on the surfaces of nano- $\mathrm{SiO}_{2}$ particles, the hydrogen bonding interaction is largely weakened, resulting in the hybrids of nano- $\mathrm{SiO}_{2} \mathrm{BenCl}$ and nano- $\mathrm{SiO}_{2} \mathrm{BenV}$ being well dispersed in the solutions with their FETEM images shown in Figures 5B and 5C. These small organic species can not only weaken the hydrogen bonding among the nano- $\mathrm{SiO}_{2}$ particles, but also change the surface of the 
nanoparticles from hydrophilic to hydrophobic; such a difference can also reduce the particleparticle interactions and benefit their dispersion in the solvents.

\section{Figure 5}

The morphologies of the MWNTs before and after they are functionalized with the - $\mathrm{BenBr}$ and other organic species have been investigated in our previous work [25]. Here, to make a comparison, one FETEM image of the MWNTP4VP hybrids is shown in Fig. 5D, from which the tubular structure of MWNTs can be clearly observed with a length up to several micrometers and diameters of about $20 \mathrm{~nm}$. When the nano- $\mathrm{SiO}_{2} \mathrm{BenCl}$ and nano- $\mathrm{SiO}_{2} \mathrm{BenV}$ hybrids are connected on the surfaces of MWNTP4VP hybrids to produce the MWNWP4VP@nano$\mathrm{SiO}_{2} \mathrm{BenCl}$ nano-cores and MWNWP4VP@nano- $\mathrm{SiO}_{2} \mathrm{BenV}$ nanocomposites, many cyclic nano$\mathrm{SiO}_{2}$ particles were observed and attached on the surfaces of the nanotubes, forming many nanoSiO $\mathrm{S}_{2}$ particle-covered $1 \mathrm{D}$ structures, though the density of the nanoparticles is somewhat low and the surfaces of the nanotubes are not fully covered, as shown in the FETEM images from Figures 5E and 5F. To give a clearer image of the hybrids, a high-resolution image of one nanotube covered with the nano- $\mathrm{SiO}_{2}$ particles is shown in Figures $5 \mathrm{G}$ and $5 \mathrm{H}$. An increase in the density of the nanoparticles on the nanotubes may require a long reaction time or more effective functionalization on the nanotubes to increase the pyridine distribution, which is currently being researched in our lab.

\subsection{Voltagramometric properties}

Fig. 6 shows the CV curves for the GC electrode covered by the casting film of nano$\mathrm{SiO}_{2} \mathrm{BenV}$ hybrids in the $0.05 \mathrm{~mol} / \mathrm{K} \mathrm{KCl}$ electrolyte solution at a scan rate of 0.1 to $0.6 \mathrm{~V} / \mathrm{s}$. One 
couple of redox waves was recorded with the cathodic potentials at about $-0.75 \sim-0.8 \mathrm{~V}$, and the anodic ones at about $-0.65 \mathrm{~V}$ (vs $\mathrm{Ag} / \mathrm{AgCl}$ ), which could be assigned to the one electron transfer of nano-SiO${ }_{2} \mathrm{BenV}^{2+} \leftrightarrow$ nano- $\mathrm{SiO}_{2} \mathrm{BenV}^{+\bullet}[37,38]$. Compared with the results in the solutions and modified electrodes of simple viologen compounds, both cathodic and anodic potentials shifted to slightly lower potentials, which may be attributed to the interfacial film resistance caused by the co-existed organics and nano- $\mathrm{SiO}_{2}$ particles. Similar phenomena have been observed for the viologen self-assembled monolayers and Langmuir-Blodgett (LB) films $[39,40]$.

Figure 6

Fig. 7A shows the $\mathrm{CV}$ curves for the $\mathrm{GC}$ electrode covered by the casting film of MWNTP4VP@nano-SiO ${ }_{2} \mathrm{BenV}$ nanocomposites in the $0.05 \mathrm{~mol} / \mathrm{l} \mathrm{KCl}$ electrolyte solution at a scan rate of 0.1 to $0.6 \mathrm{~V} / \mathrm{s}$. These curves consist of three couples of redox waves centered at about $-0.17,-0.39$, and $-0.57 \mathrm{~V}$, all of which can be designated to the electron transfer process of $\mathrm{V}^{2+} \leftrightarrow \mathrm{V}^{+\bullet}$. Three couples of redox waves suggest that they are from different kinds of viologens. From the schematic drawing of present nano-composites shown in Fig. 1, one kind of viologen is formed directly on the surface of nano- $\mathrm{SiO}_{2}$ particles, with the redox wave centered at about $-0.57 \mathrm{~V}$, which may correspond to the redox curve of nano- $\mathrm{SiO}_{2} \mathrm{BenV}$ monomers shown in Fig. 6. Another redox wave centered at $-0.39 \mathrm{~V}$ may be from the viologen substituents anchored on the surfaces of MWNTs. Finally, the redox wave centered at $-0.17 \mathrm{~V}$ may be owing to the formation of viologen dimers between two neighboring viologen units, as suggested by Buttry and co-workers in the self-assembled monolayers of viologenthiols [39]. These viologen substituents formed a thin film layer covering the nanotubes and nano- $\mathrm{SiO}_{2}$, as the carton shows 
in Fig. 7B, where viologens are anchored on the surfaces of either nano- $\mathrm{SiO}_{2}$ particles or MWNTP4VP. After reduction, viologen dimers of $\left(\mathrm{V}^{+\bullet}\right)_{2}$ were formed [39].

Besides formation of the viologen dimers in the MWNTP4VP@nano-SiO ${ }_{2} \mathrm{BenV}$ composites, a comparison of the CV curves in Fig. 7A with those in Fig. 6 indicated that the redox process of viologens became more reversible in the MWNTP4VP@nano- $\mathrm{SiO}_{2} \mathrm{BenV}$ composites. This phenomenon has been attributed to the co-existed CNTs that could act as not only a supporting layer but also an electron transfer mediator between the electrode and a redox species. The CNTs were usually functionalized with various substituents to produce positively or negatively charged surfaces that could form composite materials, LB films or layer-by-layer multilayers with electroactive species such as enzymes and viologens [41,42].

\section{Figure 7}

After being functionalized with viologens, the MWNTP4VP@nano- $\mathrm{SiO}_{2} \mathrm{BenV}$ nanocomposites were covered by positively charged organic species, which then acted as a support to interact with the negatively charged PMA clusters resulting in the formation of viologen-PMA modified nano-composites of MWNTP4VP@nano-SiO 2 BenV/PMA (Figures 1 and 7B). The electrochemical behaviors of PMA were investigated by using the casting films of MWNTP4VP@nano-SiO 2 BenV/PMA nano-composites in the $1.0 \mathrm{~mol} / \mathrm{l} \mathrm{LiCl}(\mathrm{pH}=2)$ electrolyte solution. As shown in Fig. 8A, three couples of redox waves were recorded in the potential range from -0.2 to $0.6 \mathrm{~V}$, with the cathodic potential at about $0.31,0.10,-0.10 \mathrm{~V}$ and the anodic one at $0.39,0.16,-0.02 \mathrm{~V}$, respectively, which were assigned to the successive electron transfer processes of PMA [32,43]. 


\subsection{Electrocatalytic reduction of bromate}

It has been well known that the POM-modified electrodes are good candidates for applications in electroanalysis, such as the oxidation of nitrite and the reduction of bromate $[31,44]$. Here, to illustrate the potential application of the present MWNTP4VP@nano-SiO ${ }_{2} \mathrm{BenV} / \mathrm{PMA}$ nanocomposites, we investigated the electrocatalytic reduction of bromate, as the monitoring or removal of this species is of interest due to its presence in drinking water and frequent use as a food additive [31,45].

Fig. 8A shows several $\mathrm{CV}$ curves for the GC electrode covered with the casting film of MWNTP4VP@nano-SiO ${ }_{2} \mathrm{BenV} / \mathrm{PMA}$ nano-composites in the $1.0 \mathrm{~mol} / \mathrm{l} \mathrm{LiCl}(\mathrm{pH}=2)$ in the absence and upon the addition of potassium bromate at concentrations of 1,2 , and $3 \mathrm{mmol} / \mathrm{l}$ at a scan rate of $50 \mathrm{mV} / \mathrm{s}$. These curves suggest that the cathodic current density at about $-0.08 \mathrm{~V}$ increased as the bromate concentration increased, consistent with previous research, and this has been described as a six-electron process to yield bromide $[43,46,47]$. The onset potential was related to the $\mathrm{pH}$ value of the electrolyte solution; a lower $\mathrm{pH}$ value of the electrolyte solutions benefits the electrocatalytic reaction.

\section{Figure 8}

Fig. $8 \mathrm{~B}$ is a plot of the current density at the potential of $-0.08 \mathrm{~V}$ relative to the bromate concentrations, which revealed an almost linear correction between the bromate concentrations and the current density in the concentration range from 0 to $15 \mathrm{mmol} / \mathrm{l}$. A greater increase in the bromate concentration resulted in a lower current density, that is, it did not obey the linear relationship. 


\section{Conclusions}

We have demonstrated the preparation and the electrochemical and electrocatalytic properties of nano-composites composed of functionalized $\mathrm{CNTs}$, nano- $\mathrm{SiO}_{2}$, viologens, and phosphomolybdic acid. The carbon nanotubes and nano- $\mathrm{SiO}_{2}$ were covalently connected to form nano-cores covered by layers of highly active benzyl chloride substituents, which could react with various organic species. Here, electroactive viologens were synthesized and covalently attached on the surfaces of the nano-cores, followed by the electrostatic adsorption of phosphomolybdic acid to form multi-functional nano-composites. The nano-composites modified electrodes showed well-reversible reduction-oxidation processes of the viologens and phosphomolybdic acid, as well as the efficient electrocatalytic reduction of bromate. Because both the nanotubes and nano- $\mathrm{SiO}_{2}$ particles have remarkable mechanical properties as well as high surface areas, the present nano-composites may be promising candidates for the development of chemically modified electrodes, electrochemical devices, and electrocatalytic catalysts.

Fig. 1. Schematic drawing of the preparation of the nano-hybrids of MWNTP4VP, nano$\mathrm{SiO}_{2} \mathrm{BenCl}$, and nano-SiO $\mathrm{O}_{2} \mathrm{BenV}, \mathrm{MWNTP} 4 \mathrm{VP} @$ nano- $\mathrm{SiO}_{2} \mathrm{BenCl}$ nano-cores, as well as the nanocomposites of MWNTP4VP@nano- $\mathrm{SiO}_{2} \mathrm{BenV}$ and MWNTP4VP@nano- $\mathrm{SiO}_{2} \mathrm{BenV/PMA}$. 
Fig. 2. Thermogravimetric curves for the nanomaterials of (a) nano- $\mathrm{SiO}_{2} \mathrm{BenCl}$ and (b) nano$\mathrm{SiO}_{2} \mathrm{BenV}$, (c) MWNTP4VP@nano-SiO $2 \mathrm{BenCl}$, and (d) MWNTP4VP@ nano-SiO 2 BenV from room temperature to $800{ }^{\circ} \mathrm{C}$ in air.

Fig. 3. FTIR spectra of for the nanomaterials of (a) nano- $\mathrm{SiO}_{2} \mathrm{BenCl}$ and (b) nano- $\mathrm{SiO}_{2} \mathrm{BenV}$, (c) MWNTP4VP@nano-SiO 2 BenCl, and (d) MWNTP4VP@nano-SiO 2 BenV.

Fig. 4. High-resolution XPS spectra of the MWNTP4VP@nano-SiO 2 BenV nano-composites.

Fig. 5. FETEM images of (A) nano-SiO ${ }_{2}$ particles, (B) nano- $\mathrm{SiO}_{2} \mathrm{BenCl}$, (C) nano- $\mathrm{SiO}_{2} \mathrm{BenV}$, (D) MWNTP4VP, (E) MWNTP4VP@ nanoSiO 2 BenCl, and (F) MWNTP4VP@nano-SiO ${ }_{2} \mathrm{BenV}_{\text {(G }}$ and H) High-resolution FETEM images of MWNTP4VP@ nano-SiO ${ }_{2}$ BenV.

Fig. 6. Cyclic voltammograms for the GC electrode modified with the casting films of nano$\mathrm{SiO}_{2} \mathrm{BenV}$ hybrids in the $0.05 \mathrm{~mol} / \mathrm{K} \mathrm{KCl}$ electrolyte solution at potential scan rates of 0.1 to 0.6 V/s.

Fig. 7. (A) Cyclic voltammograms for the GC electrode modified with the casting films of MWNTP4VP@nano-SiO ${ }_{2} \mathrm{BenV}$ nano-composites in the $0.05 \mathrm{~mol} / \mathrm{l} \mathrm{KCl}$ electrolyte solution at potential scan rates of 0.1 to $0.6 \mathrm{~V} / \mathrm{s}$. (B) Possible formation mechanism of the viologen dimers in the nano-composites.

Fig. 8. (A) Cyclic voltammograms for the GC electrode modified with the casting films of MWNTP4VP@nano-SiO 2 BenV/PMA nano-composites in the $1.0 \mathrm{~mol} / \mathrm{L} \mathrm{LiCl}(\mathrm{pH}=2)$

electrolyte solutions in the absence and upon the addition of bromate concentrations of 1,2 , and $3 \mathrm{mmol} / \mathrm{l}$ (from bottom to up) at a scan rate of $50 \mathrm{mV} / \mathrm{s}$. (B) Variation in the peak current density 
for bromate electrocatalytic reduction at $-0.08 \mathrm{~V}$ as a function of its concentrations at a scan rate of $50 \mathrm{mV} / \mathrm{s}$.

Table 1. Deconvolution of XPS peaks of the nano- $\mathrm{SiO}_{2} \mathrm{BenCl}$ and nano- $\mathrm{SiO}_{2} \mathrm{BenV}$ hybrids as well as the MWNTP4VP@nano-SiO 2 BenCl, MWNTP4VP@nano-SiO 2 BenV and MWNTP4VP@nano-SiO 2 BenV/PMA composites.

Acknowledgement. The authors are grateful for the National Science Foundation of China (21373058).

\section{References:}

[1] S.C. Tjong, Recent progress in the development and properties of novel metal matrix nanocomposites reinforced with carbon nanotubes and graphene nanosheets. Mater. Sci. Eng. R - Reports 74 (2013) 281-350.

[2] M.M. Barsan, M.E. Ghica, C.M.A. Brett, Electrochemical sensors and biosensors based on redox polymer/carbon nanotube modified electrodes: A review. Anal. Chim. Acta 881 (2015) $1-23$.

[3] M. Batmunkh, M.J. Biggs, J.G. Shapter, Carbon nanotubes for dye-sensitized solar cells. Small 11 (2015) 2963-2989. 
[4] Y. Chen, J. Zhang, Chemical vapor deposition growth of single-walled carbon nanotubes with controlled structures for nanodevice applications. Acc. Chem. Res. 47 (2014) 22732281.

[5] S.W. Lee, E.E.B. Campbell, Nanoelectromechanical devices with carbon nanotubes. Curr. Appl. Phys. 13 (2013) 1844-1859.

[6] G. Sakellariou, D. Priftisa D. Baskaran, Surface-initiated polymerization from carbon nanotubes: strategies and perspectives. Chem. Soc. Rev. 42 (2013) 42, 677-704.

[7] S.K. Samanta, M. Fritsch, U. Scherf, W. Gomulya, S.Z. Bisri, M.A. Loi, Conjugated polymer-assisted dispersion of single-wall carbon nanotubes: the power of polymer wrapping. Acc. Chem. Res. 47 (2014) 2446-2456.

[8] B.I. Kharisov, O.V. Kharissova, Coordination and organometallic compounds in the functionalization of carbon nanotubes. J. Coord. Chem. 67 (2014) 3769-3808.

[9] P. Bilalis, D. Katsigiannopoulos, A. Avgeropoulos, G. Sakellariou, Non-covalent functionalization of carbon nanotubes with polymers. RSC Adv. 4 (2014) 2911-2934.

[10] R. Wang, H. Wang, L. Sun, E. Wang, Y. Zhu, Y. Zhu, The fabrication and tribological behavior of epoxy composites modified by the three-dimensional polyurethane sponge reinforced with dopamine functionalized carbon nanotubes. Appl. Surf. Sci. 360 (2016) 3744.

[11] R. Hayes, A. Ahmed, T. Edge, H. Zhang, Core-shell particles: preparation, fundamentals and applications in high performance liquid chromatography. J. Chromatography A 1357 (2014) 36-52. 
[12] Y. Zhang, B.Y.W. Hsu, C.L. Ren, X. Li, J. Wang, Silica-based nanocapsules: synthesis, structure control and biomedical applications. Chem. Soc. Rev. 44 (2015) 315-335.

[13] G. Cerveau, R.J.P. Corriu, E. Framery, Nanostructured organic-inorganic hybrid materials: kinetic control of the texture. Chem. Mater. 13 (2001) 3373-3388.

[14] A. Kaushik, R. Kumar, S.K. Arya, M. Nair, B.D. Malhotra, S. Bhansali, Organic-inorganic hybrid nanocomposites-based gas sensors for environmental monitoring. Chem. Rev. 115 (2015) 4571-4606.

[15] J.G. Duque, G. Gupta, L. Cognet, B. Lounis, S.K. Doorn, A.M. Dattelbaum, New route to fluorescent single-walled carbon nanotube/silica nanocomposites: Balancing fluorescence intensity and environmental sensitivity. J. Phys. Chem. C 115 (2011) 15147-15153.

[16] Q. Fu, C. Lu, J. Liu, Selective coating of single wall carbon nanotubes with thin $\mathrm{SiO}_{2}$ layer. Nano Lett. 2 (2002) 329-332.

[17] J.C. Wojdel, S.T. Bromley, Interaction of $\mathrm{SiO}_{2}$ with single-walled carbon nanotubes. J. Phys. Chem. B 109 (2005) 1387-1391.

[18] S. Guo, J. Li, W. Ren, D. Wen, S. Dong, E. Wang, Carbon nanotube/silica coaxial nanocable as a three-dimensional support for loading diverse ultra-high-density metal nanostructures: Facile preparation and use as enhanced materials for electrochemical devices and SERS. Chem. Mater. 21 (2009) 2247-2257.

[19] Q. Li, D. Tang, J. Tang, B. Su, J. Huang, G. Chen, Carbon nanotube-based symbiotic coaxial nanocables with nanosilica and nanogold particles as labels for electrochemical immunoassay of carcinoembryonic antigen in biological fluids. Talanta 84 (2011) 538-546. 
[20] S. Guo, E. Wang, A novel sensitive solid-state electrochemiluminescence sensor material: $\mathrm{Ru}(\text { bpy })_{3}{ }^{2+}$ doped $\mathrm{SiO}_{2} @$ MWNTs coaxial nanocable. Electrochem. Commun. 9 (2007) $1252-1257$.

[21] T. Tajima, W. Sakata, T. Wada, A. Tsutsui, S. Nishimoto, M. Miyake, Y. Takaguchi, Photosensitized hydrogen evolution from water using a single-walled carbon nanotube/fullerodendron/ $\mathrm{SiO}_{2}$ coaxial nanohybrid. Adv. Mater. 23 (2011) 5750-5754.

[22] S. Musa, D.R. Rand, D.J. Cott, J. Loo, C. Bartic, W. Eberle, B. Nuttin, G. Borghs, Bottomup $\mathrm{SiO}_{2}$ embedded carbon nanotube electrodes with superior performance for integration in implantable neural microsystems. ACS Nano 6 (2012) 4615-4628.

[23] F.Z. Bouanis, C.S. Cojocaru, V. Huc, E. Norman, M. Chaigneau, J.L. Maurice, T. Mallah, D. Pribat, Direct synthesis and integration of individual, diameter-controlled single-walled nanotubes. Chem. Mater. 26 (2014) 5074-5082.

[24] Z. Sun, H. Zhang, Y. Zhao, C. Huang, R. Tao, Z. Liu, Z. Wu, Thermal-stable carbon nanotube-supported metal nanocatalysts by mesoporous silica coating. Langmuir 27 (2011) $6244-6251$.

[25] J. Liu, M. Chen, D.J. Qian, M. Liu, Fabrication of multi-pyridine functionalized carbon nanotubes as versatile coordination nano-linkers. RSC Adv. 2014, 4, 5678-5682.

[26] A.P. Duarte, M. Gressier, M.J. Menu, J. Dexpert-Ghys, J.M.A. Caiut, S.J.L. Ribeiro, Structural and luminescence properties of silica-based hybrids containing new silylateddiketonato europium(III) complex. J. Phys. Chem. C 116 (2012) 505-515. 
[27] B.K. Price, J.M. Tour, Functionalization of single-walled carbon nanotubes "on water". J. Am. Chem. Soc. 128 (2006) 12899-12904.

[28] K. Hoshina, R. Nakajima, M. Okura, Improved electrochromic performance of viologen at an ITO-nanoparticle film electrode. Appli. Surf. Sci. 313 (2014) 569-576.

[29] N.S. Lee, H.K. Shin, Y.S. Kwon, B.J. Lee, Characterization of the electrical and optical properties of viologen devices using chlorophyll a as an electron excimer. Ultramicroscopy 110 (2010) 650-654.

[30] J. Liu, M. Chen, D.J. Qian, Copper(II)-mediated layer-by-layer assembly of viologenthiolfunctionalized carbon nanotube hybrid multilayers: preparation, characterization, morphology, and electrochemical properties. Langmuir 28 (2012) 9496-9505.

[31] Y. Kim, S. Shanmugam, Polyoxometalate-reduced graphene oxide hybrid catalyst: synthesis, structure, and electrochemical properties. ACS Appl. Mater. Interfaces 5 (2013) 12197-12204.

[32] H.Y. Zhang, A.J. Miao, M. Jiang, Fabrication, characterization and electrochemistry of organic- inorganic multilayer films containing polyoxometalate and polyviologen via layerby-layer self-assembly. Mater. Chem. Phys. 141 (2013) 482-487.

[33] D. Habibi, A.R. Faraji, M. Arshadi, J.L.G. Fierro, Characterization and catalytic activity of a novel Fe nano-catalysts efficient heterogeneous catalyst for selective oxidation of ethylbenzene, cyclohexene, and benzylalcohol. J. Mol. Cat. A, Chem. 372 (2013) 90-99. 
[34] P. Singh, S. Campidelli, S. Giordani, D. Bonifazi, A. Bianco, M. Prato, Organic functionalisation and characterisation of single-walled carbon nanotubes. Chem. Soc. Rev. 38 (2009) 2214-2230.

[35] T. Ramanathan, F.T. Fisher, R.S. Ruoff, L.C. Brinson, Amino-functionalized carbon nanotubes for binding to polymers and biological systems. Chem. Mater. 17 (2005) 12901295.

[36] R.K. Sharma, S. Sharma, Silica nanosphere-supported palladium(II) furfural complex as a highly efficient and recyclable catalyst for oxidative amination of aldehydes. Dolton Trans. 43 (2014) 1292-1304.

[37] B. Cheng, A.E. Kaifer, Electrochemistry of viologen dications in cholate media and competition between the cholate assemblies and the cucurbit[7]uril host. Langmuir 31 (2015) 2997-3002.

[38] T. Higashi, T. Sagara, Diphenyl viologen on an HOPG electrode surface: less sharp redox wave than dibenzyl viologen. Langmuir 29 (2013) 11516-11524.

[39] X. Tang, T.W. Schneider, J.W. Walker, D.A. Buttry, Dimerized $\pi$-complexes in selfassembled monolayers containing viologens: an origin of unusual wave shapes in the voltammetry of monolayers. Langmuir 12 (1996) 5921-5933.

[40] S.S. Zhang, H.L. Wang, M. Chen, D.J. Qian, Monolayers and Langmuir-Blodgett films of $\mathrm{Fe}^{2+}$-mediated polyelectrolyte with viologen derivatives as linkers at the air-water interface. Colloids Surf. A: Physicochem. Eng. Aspects 384 (2011) 561- 569. 
[41] M. Alvaro, C. Aprile, P. Atienzar, H. Garcia, Preparation and photochemistry of single wall carbon nanotubes having covalently anchored viologen units. J. Phys. Chem. B 109 (2005) $7692-7697$.

[42] X. Wang, H.X. Huang, A.R. Liu, B. Liu, T. Wakayama, C. Nakamura, J. Miyake, D.J. Qian, Layer-by-layer assembly of single-walled carbon nanotube-poly(viologen) derivative multilayers and their electrochemical properties. Carbon 44 (2006) 2115-2121.

[43] L. Ding, Y. Liu, S.X. Guo, J. Zhai, A.M. Bond, J. Zhang, Phosphomolybdate@poly(diallyldimethylammonium chloride)-reduced graphene oxide modified electrode for highly efficient electrocatalytic reduction of bromate. J. Electroanal. Chem. 727 (2014) 69-77.

[44] A. Dolbecq, E. Dumas, C.R. Mayer, P. Mialane, Hybrid organic-inorganic polyoxometalate compounds: from structural diversity to applications. Chem. Rev. 110 (2010) 6009-6048.

[45] M.M. Moore, T. Chen, Mutagenicity of bromate: Implications for cancer risk assessment. Toxicology 221 (2006) 190-196.

[46] M. Skunik, P.J. Kulesz, Phosphomolybdate-modified multi-walled carbon nanotubes as effective mediating systems for electrocatalytic reduction of bromate. Anal. Chim. Acta 631 (2009) 153-160.

[47] S.S. Hassan, Y. Liu, Sirajuddin, A.R. Solangi, A.M. Bond, J. Zhang, Phosphomolybdatedoped-poly(3,4-ethylenedioxythiophene) coated gold nanoparticles: Synthesis, characterization and electrocatalytic reduction of bromate. Anal. Chim. Acta 803 (2013) 4146. 
Table 1

\begin{tabular}{|c|c|c|c|c|c|c|c|c|c|}
\hline \multirow[b]{2}{*}{ Material } & \multicolumn{9}{|c|}{ Detected Element } \\
\hline & $\operatorname{Br}(3 d)$ & $\operatorname{Si}(2 p)$ & $\mathrm{P}(3 \mathrm{p})$ & $\mathrm{Cl}(2 \mathrm{p})$ & $\operatorname{Mo}(3 d)$ & $\mathrm{C}(1 \mathrm{~s})$ & $\mathrm{N}(1 \mathrm{~s})$ & $\mathrm{O}(1 \mathrm{~s})$ & $\mathrm{I}(3 \mathrm{~d})$ \\
\hline nano- $\mathrm{SiO}_{2} \mathrm{BenCl}$ & & 103.8 & & 197.8 & & 284.6 & & 533.2 & \\
\hline nano- $\mathrm{SiO}_{2} \mathrm{BenV}$ & & 103.8 & & 198.0 & & 284.6 & 400.0 & 533.4 & $619.8 / 631.8$ \\
\hline $\begin{array}{c}\text { MWNTP4VP@ nano- } \\
\mathrm{SiO}_{2} \mathrm{BenCl}\end{array}$ & 69.8 & 103.8 & & 198.4 & & 284.6 & 399.2 & 533.4 & \\
\hline $\begin{array}{l}\text { MWNTP4VP@ nano- } \\
\mathrm{SiO}_{2} \mathrm{BenV}\end{array}$ & 70.0 & 103.6 & & 199.2 & & 284.6 & 398.8 & 533.4 & $618.6 / 630.2$ \\
\hline $\begin{array}{c}\text { MWNTP4VP@ nano- } \\
\mathrm{SiO}_{2} \text { BenV/PMA }\end{array}$ & & 103.5 & 133.0 & 198.4 & $232.0 / 235.0$ & 284.6 & 399.2 & 533.2 & \\
\hline
\end{tabular}


Fabrication, electrochemical and electrocatalytic properties of carbon nanotube@nano-SiO ${ }_{2}$ BenV/phosphomolybdic acid polynary nanocomposite

\title{
materials
}

\author{
Jiang Liu, ${ }^{a}$ Jing Wang, ${ }^{a}$ Wen-Bo Wang, ${ }^{a}$ Meng Chen, ${ }^{b}$ Dong-Jin Qian ${ }^{a *}$ \\ ${ }^{a}$ Department of Chemistry, Fudan University, 220 Handan Road, Shanghai 200433, China \\ ${ }^{b}$ Department of Material Science, Fudan University, 220 Handan Road, Shanghai 200433,
}

China 


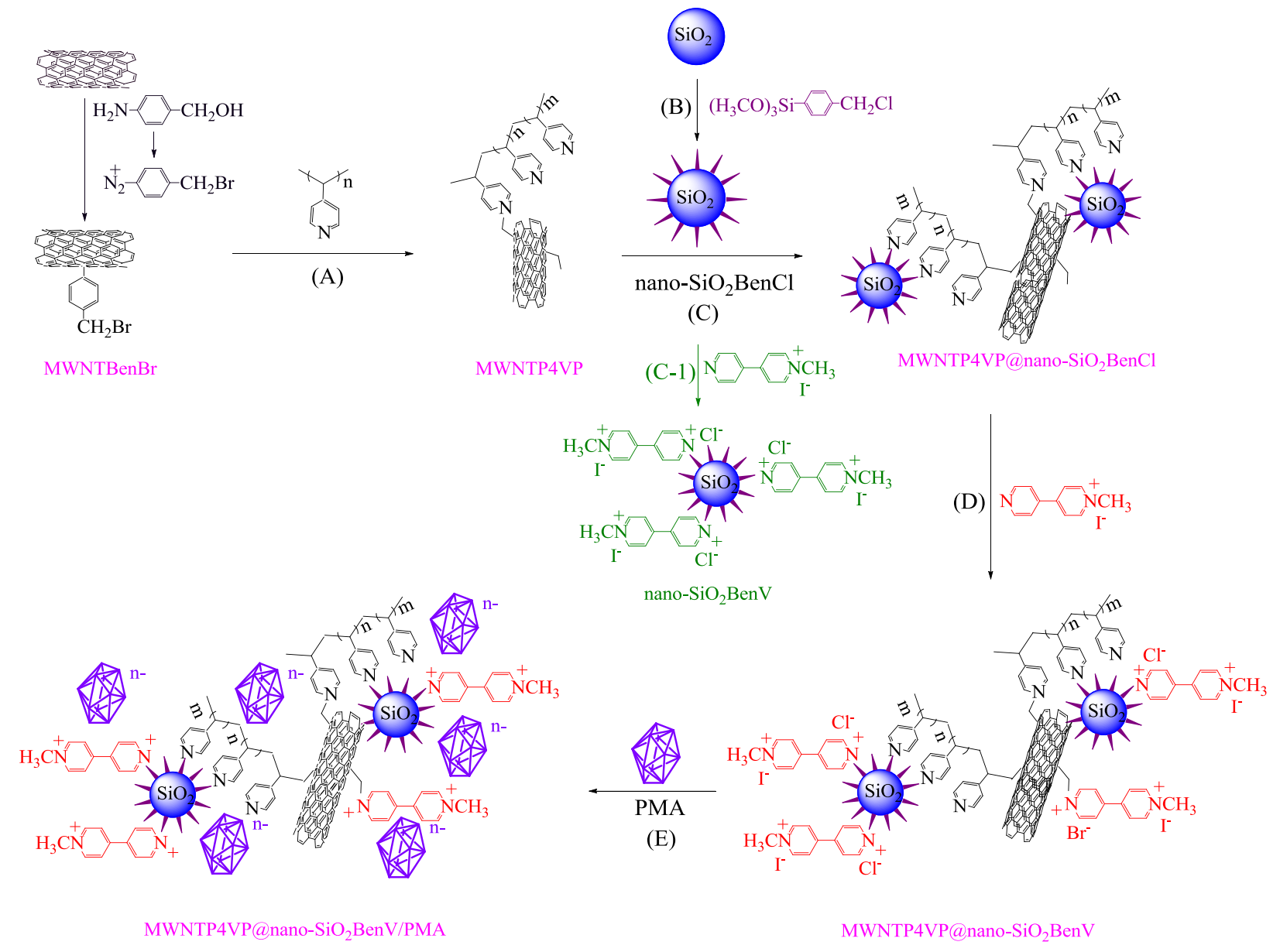

Figure 1 


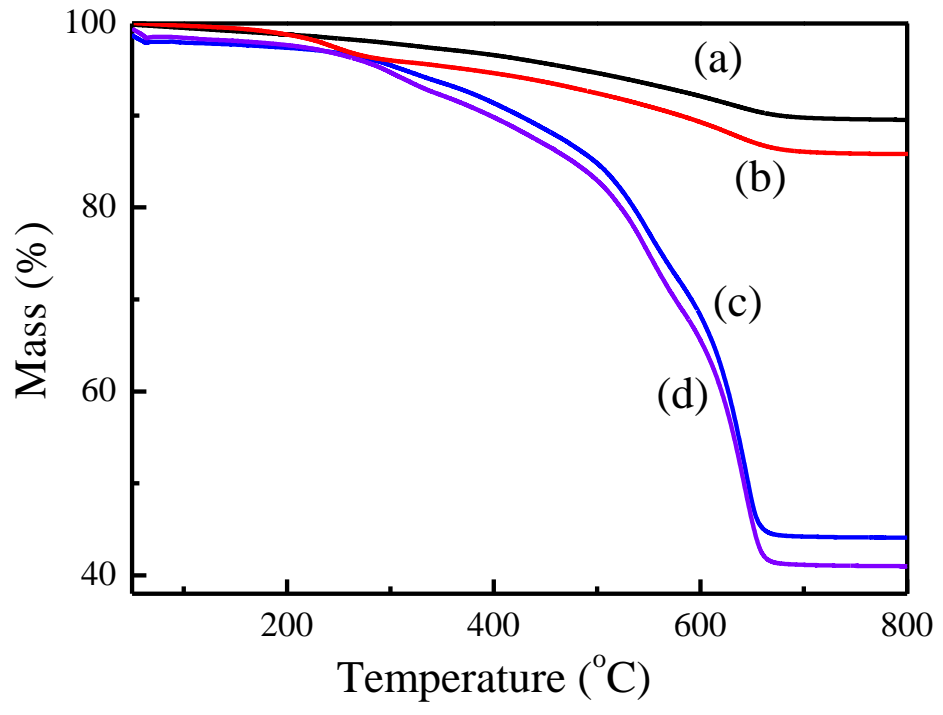

Figure 2 


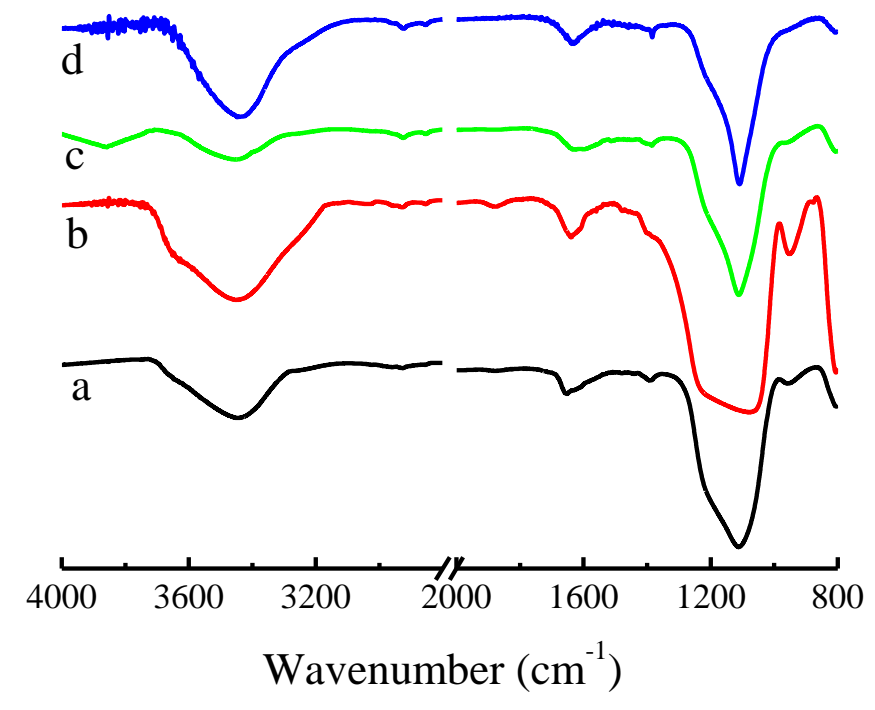

Figure 3 


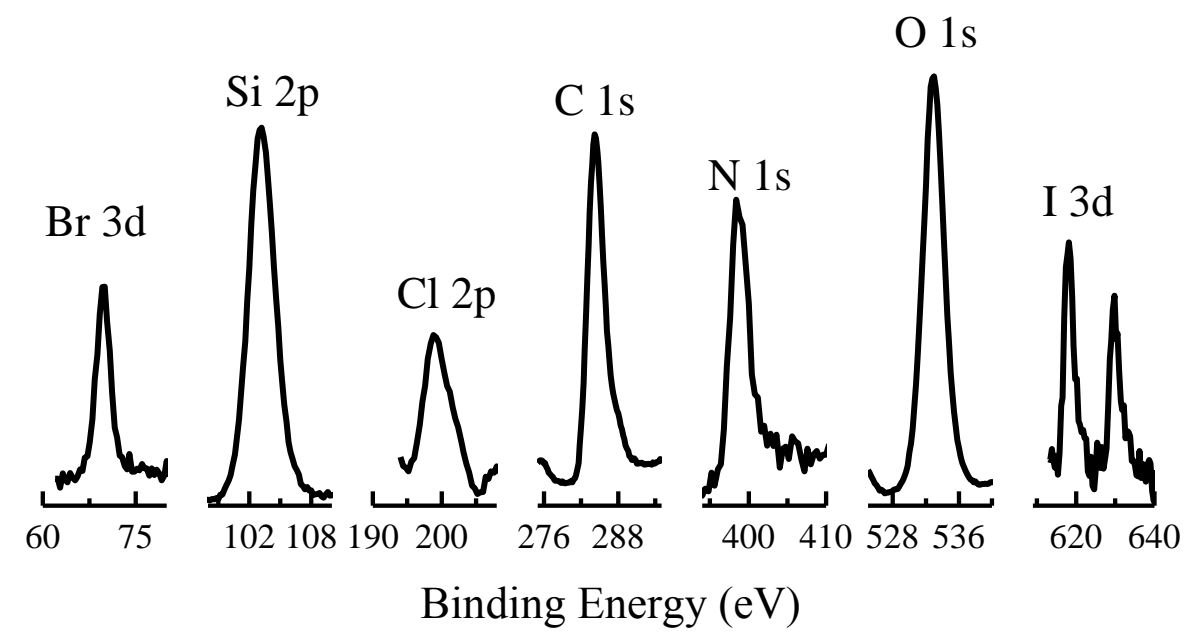

Figure 4 

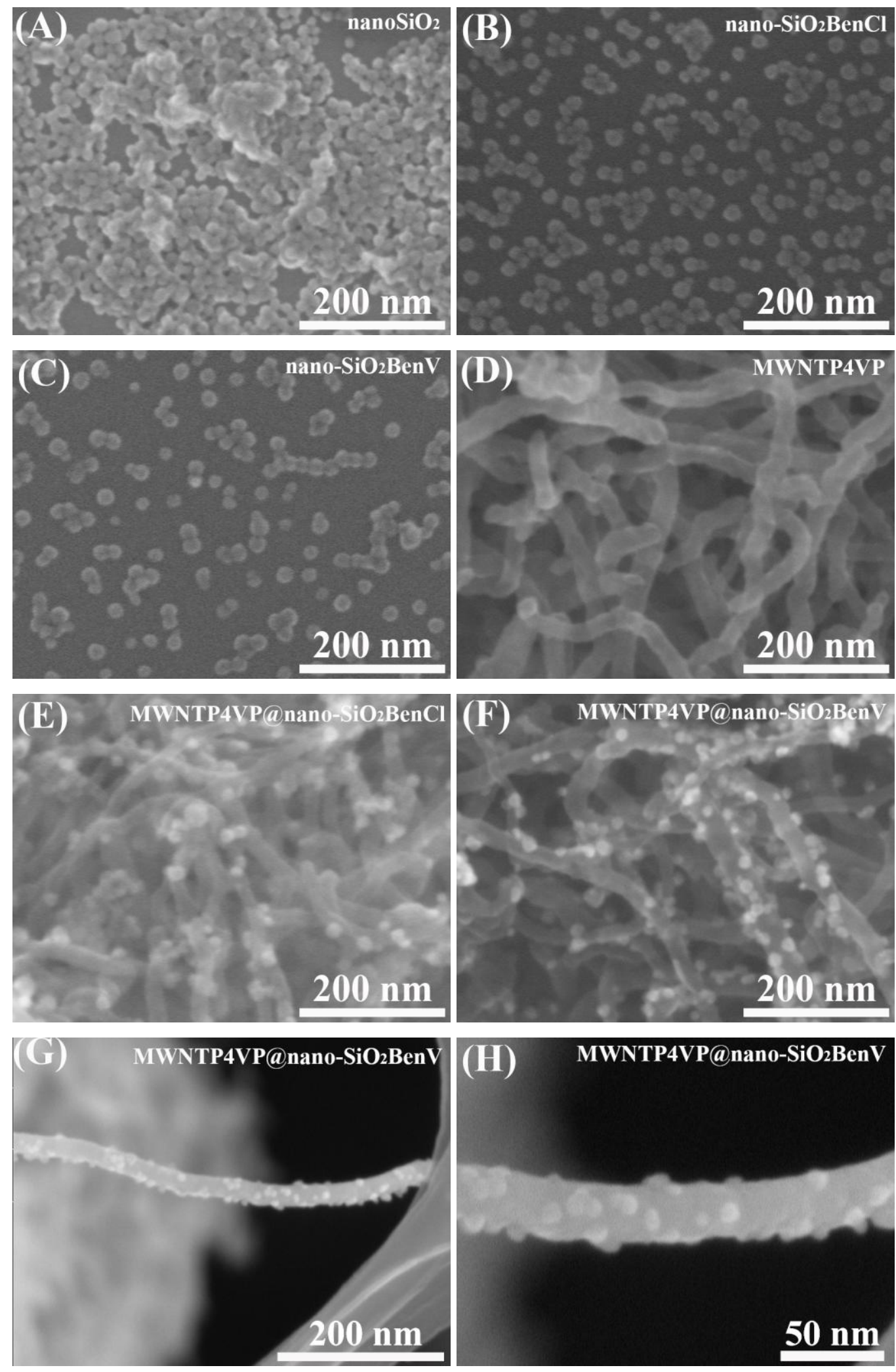

Figure 5 


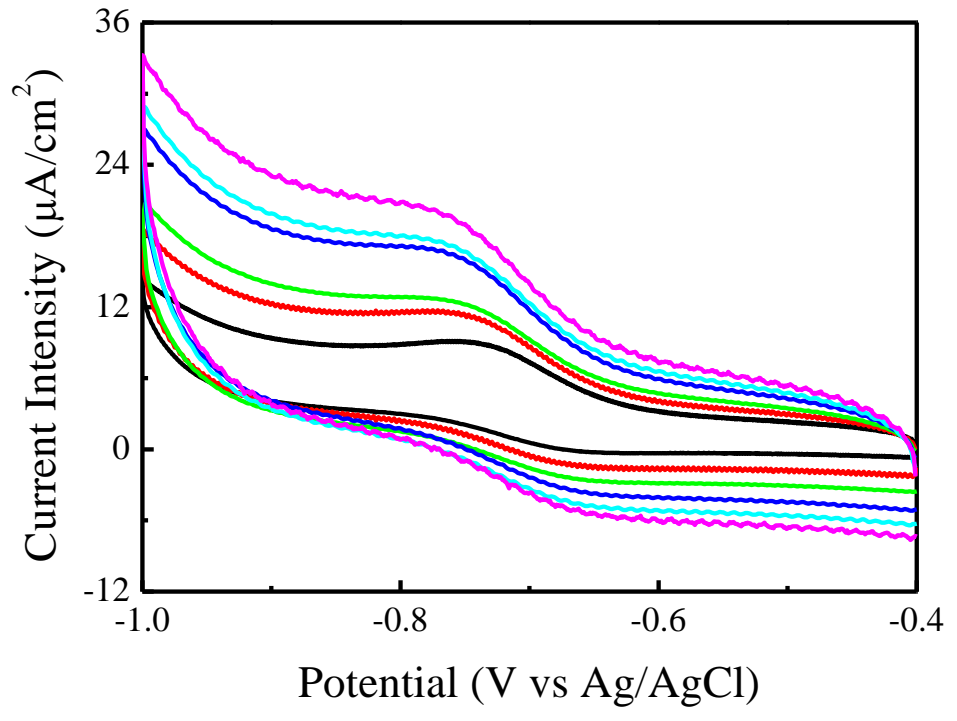

Figure 6 

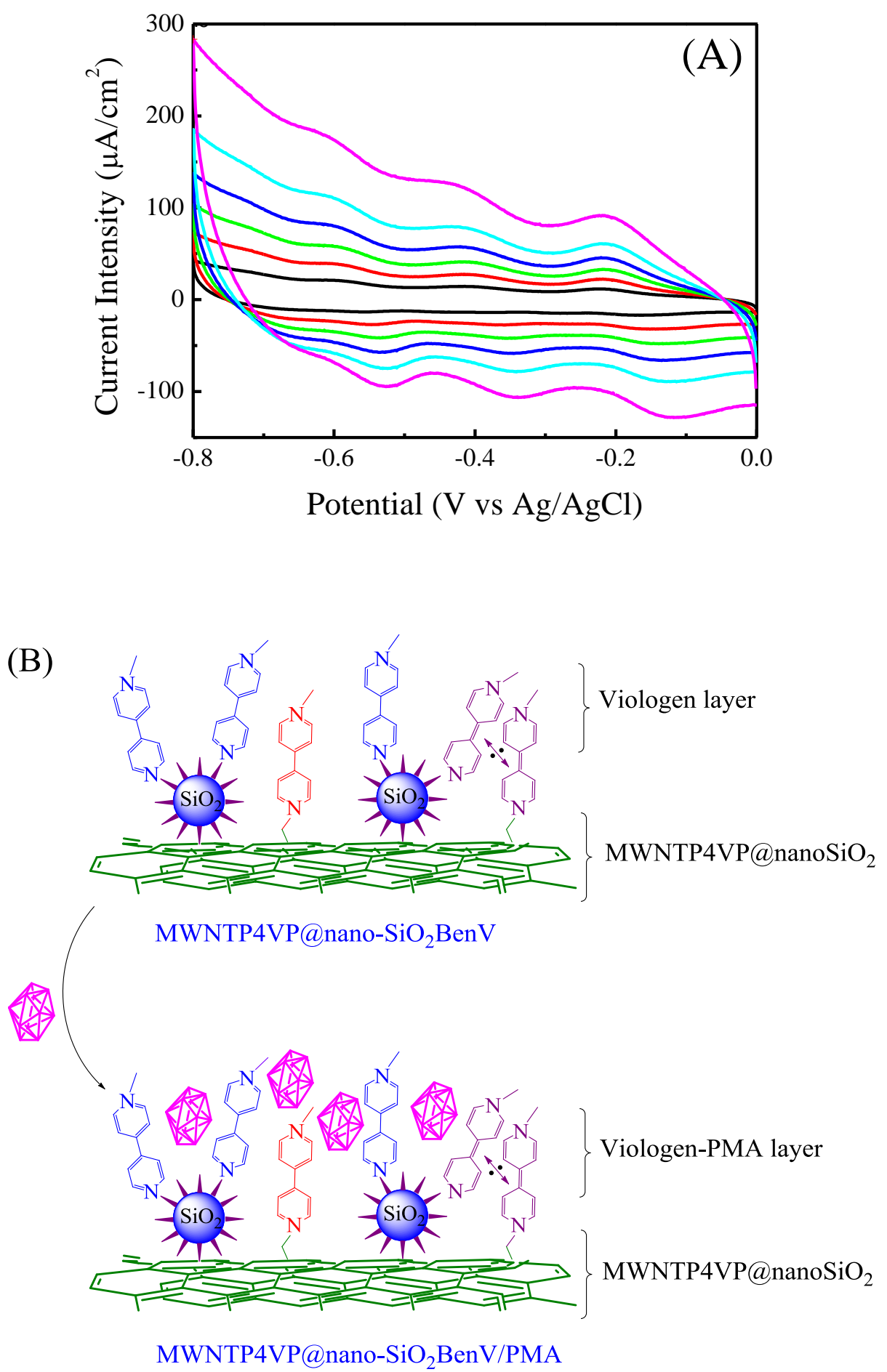

Figure 7 

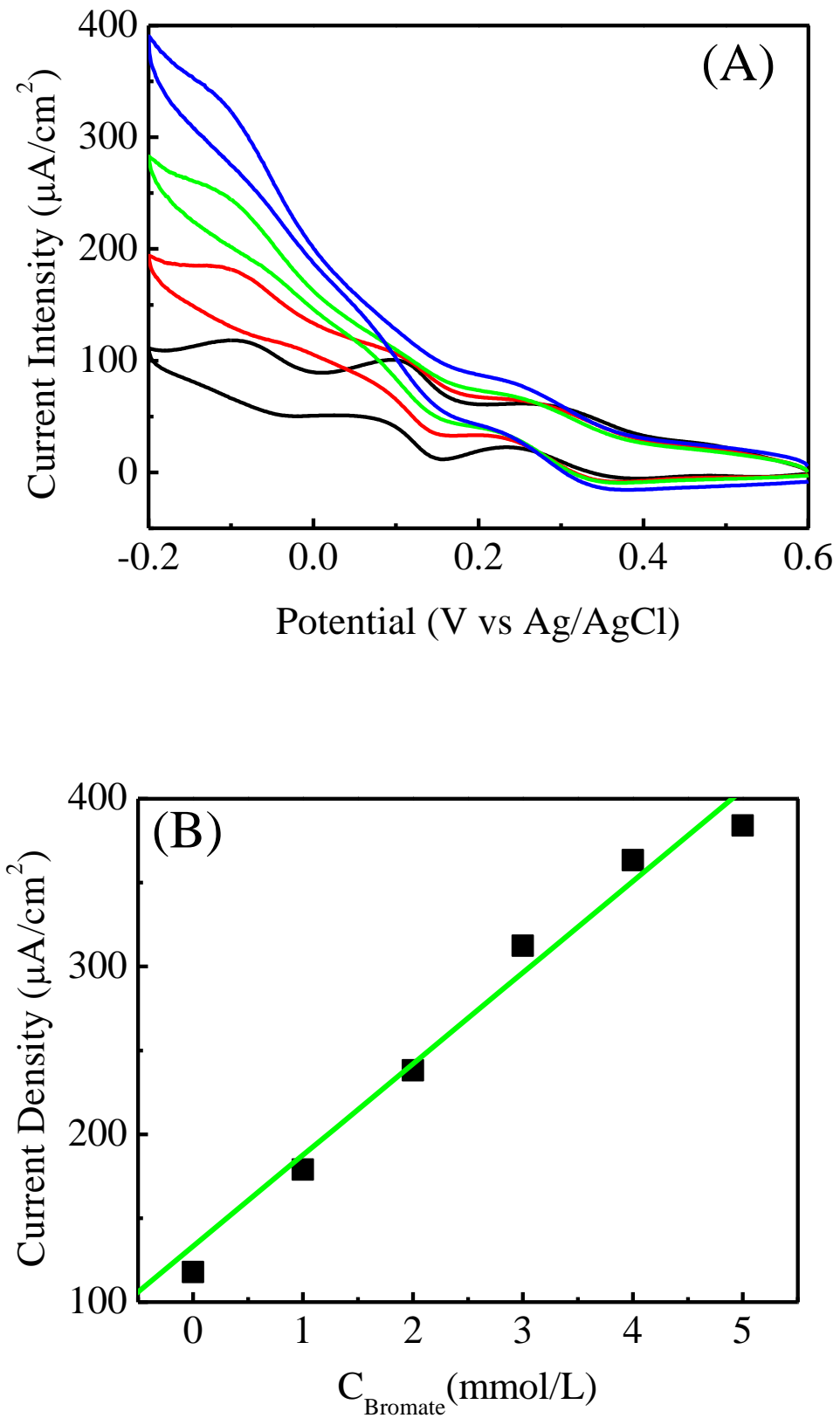

Figure 8 
Fabrication, electrochemical and electrocatalytic properties of carbon nanotube@nano-SiO $\mathrm{S}_{2} \mathrm{BenV} / \mathrm{phosphomolybdic} \mathrm{acid} \mathrm{polynary} \mathrm{nanocomposite}$ materials

Jiang Liu, ${ }^{a}$ Jing Wang, ${ }^{a}$ Wen-Bo Wang, ${ }^{a}$ Meng Chen, ${ }^{b}$ Dong-Jin Qian ${ }^{{ }^{*}}$

${ }^{a}$ Department of Chemistry, Fudan University, 220 Handan Road, Shanghai 200433, China

${ }^{b}$ Department of Material Science, Fudan University, 220 Handan Road, Shanghai 200433,

China
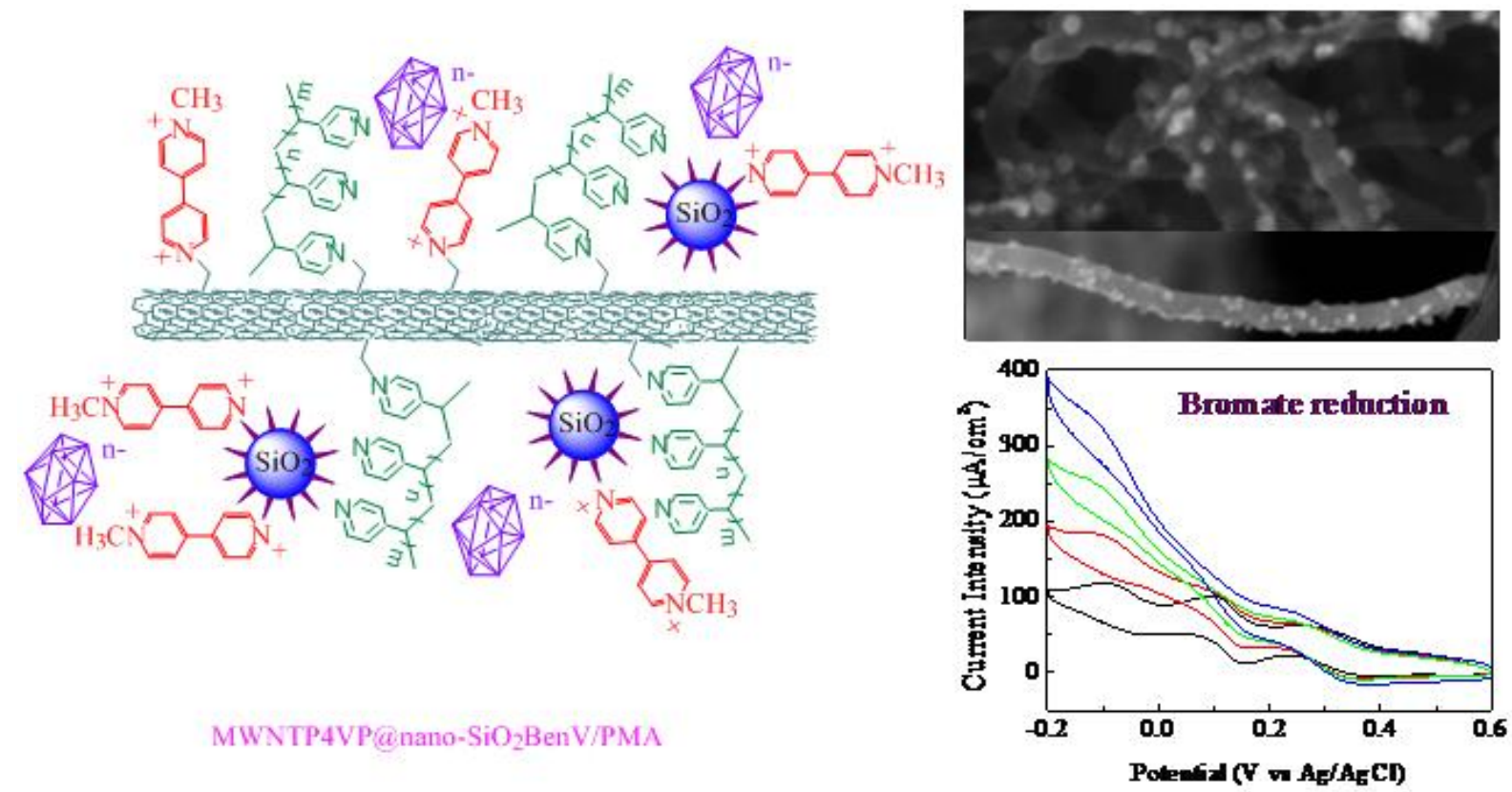